\title{
Blood urea nitrogen in the prediction of in-hospital mortality of patients with acute aortic dissection
}

\author{
Jun Liu ${ }^{1}$, Lian-Lian Sun ${ }^{1}$, Jue Wang ${ }^{2}$, Guang $\mathrm{Ji}^{1}$ \\ ${ }^{1}$ Division of Cardiology, the First Affiliated Hospital of Wenzhou Medical University, \\ Wenzhou, Zhejiang, P. R. China \\ ${ }^{2}$ Division of Cardiothoracic Surgery, the First Affiliated Hospital of \\ Wenzhou Medical University, Wenzhou, Zhejiang, P. R. China
}

\begin{abstract}
Background: Blood urea nitrogen (BUN) has been shown to be associated with adverse cardiovascular disease outcomes. The aim of the present study was to evaluate the prognostic role of BUN in patients with acute aortic dissection (AAD). Hypothesis: BUN has correlation with in-hospital mortality of patients with $A A D$.

Methods: Patients admitted to the emergency room within the first $24 \mathrm{~h}$ of onset of $A A D$ were included in the study. BUN levels were measured on admission and the endpoints were mortality during hospitalization after receiving surgical or endovascular repair.

Results: A total of 192 patients with AAD were enrolled. During hospitalization, 19 patients died and 173 patients survived. Increased levels of BUN (8.9 [7.0-9.7] vs. 6.0 [5.1-7.2] mmol/L, $p<0.001)$ were found in non-survivors compared with those survived. Using multivariable logistic analysis, $B U N$ was an independent predictor of in-hospital mortality in patients with $A A D(O R$ 1.415, 95\% CI 1.016-1.971, $p=0.040$ ). Furthermore, using receiver operating characteristic analysis, the optimal cutoff value for BUN was $6.95 \mathrm{mmol} / \mathrm{L}$. Under this value, the area under the curve was $0.785(95 \% \mathrm{CI}$ $0.662-0.909, p<0.001)$ and the sensitivity and specificity to predict in-hospital mortality was $78.9 \%$, and $72.2 \%$, respectively.
\end{abstract}

Conclusions: Admission BUN levels were an independent predictor for in hospital mortality in patients with $A A D$. (Cardiol J 2018; 25, 3: 371-376)

Key words: blood urea nitrogen, acute aortic dissection, in-hospital mortality

\section{Introduction}

The relationship between renal dysfunction and adverse cardiovascular disease outcomes have been well established in patients with coronary artery disease (CAD) [1] and in those with heart failure [2]. Prior studies utilizing serum creatinine, estimates of glomerular filtration rate (eGFR) or blood urea nitrogen (BUN) to investigate the prognostic role of renal dysfunction in cardiovascular disease have found that $\mathrm{BUN}$ is more sensitive than creatinine and eGFR in predicting poor clinical outcomes [1, 3, 4]. In fact, BUN has also been demonstrated to be a valid biomarker for disease severity and prognosis in many other conditions such as communityacquired pneumonia [5], acute pancreatitis [6] and acute intracerebral hemorrhage [7].

Acute aortic dissection (AAD) is a life-threatening disorder that necessitates immediate management. Previous studies have demonstrated that kidney injury is a risk factor for both short and long term mortality in patients with $\mathrm{AAD}[8,9]$. However, these studies used serum creatinine

Address for correspondence: Jun Liu, MD, Division of Cardiology, the First Affiliated Hospital of Wenzhou Medical University, Wenzhou, Zhejiang, P. R. China, tel: +86577 55579233, e-mail: liujunemail1@163.com 
as a surrogate marker for renal dysfunction and whether BUN could also provide some prognostic significance in these patients is unknown. Hence, the aim of the present study was to evaluate the relationship between BUN and in hospital mortality in patients admitted with AAD.

\section{Methods}

\section{Study population}

Herein is retrospectively investigated the medical records of patients with AAD admitted to the First Affiliated Hospital of Wenzhou Medical University between December 2012 and June 2016. The study was reviewed and approved by the ethics committee of the hospital and informed consent was waived due to its retrospective nature.

The diagnosis of AAD was confirmed by multidetector computed tomography scan and the type of AAD was classified in accordance with Stanford University criteria. Patients were included in the present study if they fulfilled the following criteria: (1) the time interval between symptoms onset and hospital admission of $\leq 24 \mathrm{~h}$; (2) receiving surgical or endovascular repair for AAD during hospitalization. Exclusion criteria included: (1) presence of AAD for more than $24 \mathrm{~h}$; (2) diagnosis with Marfan syndrome; (3) prior history of aortic dissection (AD).

\section{Treatment}

For patients with confirmed diagnosis of AD, urapidil, sodium nitroprusside or nitroglycerine were was administered intravenously to reduce systolic blood pressure (SBP) to $100-120 \mathrm{mmHg}$. Beta-blocker was administered to all patients except those with the contraindication.

All type A AD patients and a small part of type $B A D$ patients underwent surgery repair via cardiopulmonary bypass. Endovascular repair was performed using commercially available endografts with the patients under general anesthesia.

\section{Data collection}

The following clinical data were collected on admission: age, sex, presence of hypertension, CAD, dyslipidemia, diabetes mellitus (DM), smoking and drinking habits, type of $\mathrm{AAD}$, aortic diameter, blood pressure, heart rate, and laboratory data.

\section{Endpoint}

The study endpoint was defined as all-cause mortality during hospitalization.

\section{Statistical analysis}

Normality of continuous data was assessed using the Shapiro-Wilk test. Continuous variables are presented as the mean \pm standard deviation or median and interquartile range according to whether they follow normal distributions. Categorical variables are presented as proportions. Comparisons between groups were performed with unpaired Student $t$ tests for normally distributed continuous variables and Wilcoxon Mann-Whitney tests for non-normally distributed continuous variables. Categorical variables were compared by $\chi^{2}$ tests or Fisher's exact test when appropriate. Univariate analysis and multiple logistic regression analysis were used to identify the predictors of in-hospital mortality. Receiver operating characteristic (ROC) analysis was performed to determine the cut-off value for BUN in predicting inhospital mortality with high sensitivity and specificity. A p value $<0.05$ was considered to be statistically significant. All the statistical analyses were performed using SPSS statistical software, version 19.0 (SPSS Inc., Chicago, Illinois).

\section{Results}

\section{Baseline clinical characteristics}

Six hundred twenty six patients were identified with a diagnosis of $\mathrm{AD}$, of whom 251 were admitted within $24 \mathrm{~h}$ after onset of symptoms. Among them, 8 patients diagnosed with Marfan syndrome, 9 patients with prior history of $\mathrm{AD}$ and 42 patients died during hospitalization with conservative treatment were excluded from analysis. A total of 192 patients met our eligibility criteria and were included in the present study. During hospitalization, 19 patients died and 173 patients survived.

Patient characteristics are shown in Table 1. There were no significant differences in sex, hypertension, CAD, dyslipidemia, DM, smoking, alcohol use, aortic diameter, heart rate and neutrophil counts between the two groups. Compared with survivors, the non-survivors were older (62 [50-66] vs. 50 [44-60] years, $p=0.03$ ), had a higher percentage of type A AD $(78.9 \%$ vs. $43.9 \%, \mathrm{p}=0.003)$. The non-survivors had a significantly lower SBP $(116.2 \pm 26.0$ vs. $145.6 \pm$ $\pm 26.0 \mathrm{mmHg}, \mathrm{p}<0.001)$, diastolic blood pressure (DBP; $66.6 \pm 15.5$ vs. $80.2 \pm 18.6 \mathrm{mmHg}$, $\mathrm{p}=0.003)$, and hemoglobin (122 [111-133] vs. $132[122-144] \mathrm{g} / \mathrm{L}, \mathrm{p}=0.006)$ on admission. In addition, white blood cell counts (14.8 [13.1-18.0] vs. $\left.13.2[11.2-15.8] \times 10^{9} / \mathrm{L}, \mathrm{p}=0.047\right)$, creatinine 
Table 1. Baseline characteristics of the patients.

\begin{tabular}{|c|c|c|c|c|}
\hline Variable & All patients $(n=192)$ & Survivor $(n=173)$ & Non-survivor $(n=19)$ & $\mathbf{P}$ \\
\hline Age [years] & $51(44-62)$ & $50(44-60)$ & $62(50-66)$ & 0.03 \\
\hline Male & $151(78.6 \%)$ & $135(78.0 \%)$ & $16(84.2 \%)$ & 0.388 \\
\hline Hypertension & $137(71.4 \%)$ & $122(70.5 \%)$ & $15(78.9 \%)$ & 0.316 \\
\hline Coronary artery disease & $1(0.5 \%)$ & $1(0.6 \%)$ & $0(0 \%)$ & 0.901 \\
\hline Dyslipidemia & $1(0.5 \%)$ & $1(0.6 \%)$ & $0(0 \%)$ & 0.901 \\
\hline Diabetes mellitus & $7(3.6 \%)$ & $6(3.5 \%)$ & $1(5.3 \%)$ & 0.524 \\
\hline Smoking & $66(34.4 \%)$ & $61(35.3 \%)$ & $5(26.3 \%)$ & 0.612 \\
\hline Alcohol use & $41(21.4 \%)$ & $39(22.5 \%)$ & $2(10.5 \%)$ & 0.181 \\
\hline Type A AD & $91(47.4 \%)$ & $76(43.9 \%)$ & $15(78.9 \%)$ & 0.003 \\
\hline $\mathrm{SBP}[\mathrm{mmHg}]$ & $142.7 \pm 27.4$ & $145.6 \pm 26.0$ & $116.2 \pm 26.0$ & $<0.001$ \\
\hline $\mathrm{DBP}[\mathrm{mmHg}]$ & $78.8 \pm 18.7$ & $80.2 \pm 18.6$ & $66.6 \pm 15.5$ & 0.003 \\
\hline Aortic diameter [mm] & $39(36-43)$ & $39(36-43)$ & $41(35-45)$ & 0.329 \\
\hline Heart rate $[\mathrm{bpm}]$ & $80.9 \pm 16.6$ & $80.5 \pm 16.3$ & $84.4 \pm 19.0$ & 0.333 \\
\hline White blood cell $\left[\times 10^{9} / L\right]$ & $13.5(11.2-16.0)$ & $13.2(11.2-15.8)$ & $14.8(13.1-18.0)$ & 0.047 \\
\hline Neutrophil $\left[\times 10^{9} / \mathrm{L}\right]$ & $12.0 \pm 3.7$ & $11.8 \pm 3.6$ & $13.3 \pm 4.3$ & 0.100 \\
\hline Hemoglobin [g/L] & $132(122-144)$ & $132(122-144)$ & $122(111-133)$ & 0.006 \\
\hline Creatinine $[\mathrm{mmol} / \mathrm{L}]$ & $78.0(64.0-102.8)$ & $76.0(63.0-96.0)$ & $127.0(83.0-150.0)$ & $<0.001$ \\
\hline BUN [mmol/L] & $6.1(5.2-7.6)$ & $6.0(5.1-7.2)$ & $8.9(7.0-9.7)$ & $<0.001$ \\
\hline
\end{tabular}

Data are expressed as mean \pm standard deviation, median (25th-75th percentile) or the number (percentages) of patients. The bold values indicate statistical significance; AD - aortic dissection; BUN — blood urea nitrogen; DBP — diastolic blood pressure; SBP — systolic blood pressure

Table 2. Univariable logistic regression for in-hospital mortality.

\begin{tabular}{lccc}
\hline Variable & OR & 95\% Cl & P \\
\hline Age & 1.037 & $0.999-1.075$ & $\mathbf{0 . 0 0 5 4}$ \\
Type A AD & 4.786 & $1.526-15.011$ & $\mathbf{0 . 0 0 7}$ \\
SBP [mmHg] & 0.957 & $0.929-0.976$ & $<\mathbf{0 . 0 0 1}$ \\
DBP [mmHg] & 0.960 & $0.934-0.987$ & $\mathbf{0 . 0 0 4}$ \\
White blood cell [×10\%/L] & 1.116 & $0.997-1.249$ & 0.057 \\
Hemoglobin [g/L] & 0.965 & $0.940-0.992$ & $\mathbf{0 . 0 1 0}$ \\
Creatinine [mmol/L] & 1.005 & $1.000-1.010$ & $\mathbf{0 . 0 3 7}$ \\
BUN [mmol/L] & 1.330 & $1.118-1.582$ & $\mathbf{0 . 0 0 1}$ \\
\hline
\end{tabular}

$\mathrm{Cl}$ - confidence interval; OR — odds ratio; rest abbreviations as in Table 1

(127.0 [83.0-150.0] vs. 76.0 [63.0-96.0] mmol/L, $\mathrm{p}<0.001)$ and BUN (8.9 [7.0-9.7] vs. 6.0 [5.1-7.2] $\mathrm{mmol} / \mathrm{L}, \mathrm{p}<0.001$ ) levels were higher in the nonsurvivor group than in the survivor group.

\section{Predictors for in-hospital mortality}

Logistic regression was performed to identify potential predictors for in-hospital mortality in patients with AAD. Variables included in the univariable logistic regression analysis for inhospital mortality were age, type A AD, SBP, DBP, white blood cell counts, hemoglobin, creatinine and BUN. Except for white blood cell counts, all variables included were significantly associated with in-hospital mortality in univariable logistic regression (Table 2). The multivariable logistic regression model for in-hospital mortality included all variables from the univariable analysis. The only variables that remained as independent predictors of in-hospital mortality were age (odds ratio [OR] $1.059,95 \%$ confidence interval [CI] 1.003-1.119, $\mathrm{p}=0.039)$, SBP (OR 0.962, 95\% CI 0.928-0.998, 
Table 3. Multivariable logistic regression for in-hospital mortality.

\begin{tabular}{lccc}
\hline Variable & OR & $95 \% \mathrm{Cl}$ & P \\
\hline Age & 1.059 & $1.003-1.119$ & $\mathbf{0 . 0 3 9}$ \\
Type A AD & 2.099 & $0.451-9.774$ & 0.345 \\
SBP [mmHg] & 0.962 & $0.928-0.998$ & $\mathbf{0 . 0 3 7}$ \\
DBP [mmHg] & 1.000 & $0.955-1.047$ & 0.992 \\
White blood cell $\left[\times 10^{9} / \mathrm{L}\right]$ & 1.113 & $0.946-1.310$ & 0.196 \\
Hemoglobin $[\mathrm{g} / \mathrm{L}]$ & 0.983 & $0.943-1.026$ & 0.435 \\
Creatinine $[\mathrm{mmol} / \mathrm{L}]$ & 0.996 & $0.984-1.007$ & 0.469 \\
BUN $[\mathrm{mmol} / \mathrm{L}]$ & 1.415 & $1.016-1.971$ & $\mathbf{0 . 0 4 0}$ \\
\hline
\end{tabular}

$\mathrm{Cl}$ - confidence interval; OR - odds ratio; rest abbreviations as in Table 1.

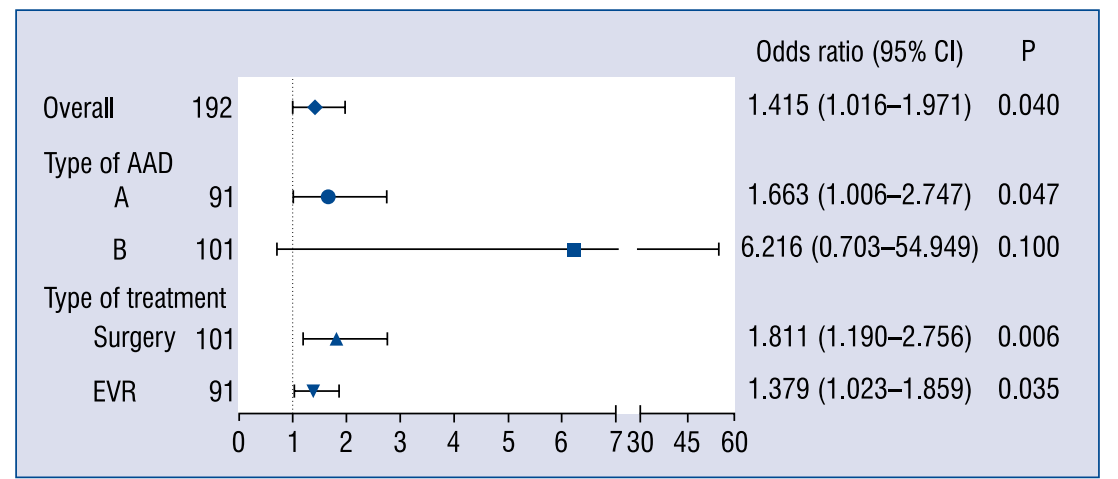

Figure 1. Odds ratios of blood urea nitrogen for predicting in-hospital mortality according to the type of acute aortic dissection (AAD) and treatment; $\mathrm{Cl}$ - confidence interval; EVR — endovascular repair.

Table 4. Diagnostic value of blood urea nitrogen for in-hospital mortality.

\begin{tabular}{ccccccc}
\hline AUC & Cut-off value & SE & $95 \% \mathrm{Cl}$ & $\mathrm{P}$ & Sensitivity & Specificity \\
\hline 0.785 & 6.95 & 0.063 & $0.662-0.909$ & $<0.001$ & 0.789 & 0.722 \\
\hline
\end{tabular}

AUC — area under the curve; $\mathrm{Cl}$ - confidence interval; SE — standard error

$\mathrm{p}=0.0037)$ and BUN (OR 1.415, 95\% CI 1.016$-1.971, \mathrm{p}=0.040$ ) (Table 3).

Given the high mortality rates in patients with type $\mathrm{A} \mathrm{AD}$ and those who undergo surgery treatment, subgroup analysis was performed by the type of $\mathrm{AD}$ and treatment. In patients with type A AD, BUN was associated with high risk of in-hospital mortality (OR 1.663, 95\% CI 1.006-2.747, $\mathrm{p}=0.047)$. For patients with type $\mathrm{B} A D, B U N$ was not an independent predictor for in-hospital mortality. As for the subgroup, stratified by surgical or endovascular repair, BUN independently predicted in-hospital mortality (OR 1.811, 95\% CI 1.190-2.756, $\mathrm{p}=0.006 ;$ OR $1.379,95 \%$ CI $1.023-1.859$, $\mathrm{p}=0.035$, respectively) (Fig. 1).

\section{ROC analysis}

ROC analysis yielded an area under the curve (AUC) statistic of 0.785 (95\% CI 0.662-0.909, $\mathrm{p}<0.001$ ) and the cut-off value of BUN to predict in-hospital mortality was 6.95 (sensitivity $78.9 \%$, specificity $72.2 \%$ ) (Table 4, Fig. 2).

\section{Discussion}

The main findings of the present study were that admission BUN levels were independently associated with in-hospital mortality in patients with $\mathrm{AAD}$ admitted within $24 \mathrm{~h}$ after onset of symptoms. When BUN was $\geq 6.95 \mathrm{mmol} / \mathrm{L}$, the sensitivity and specificity for in-hospital mortality were $78.9 \%$ and $72.2 \%$, respectively. 


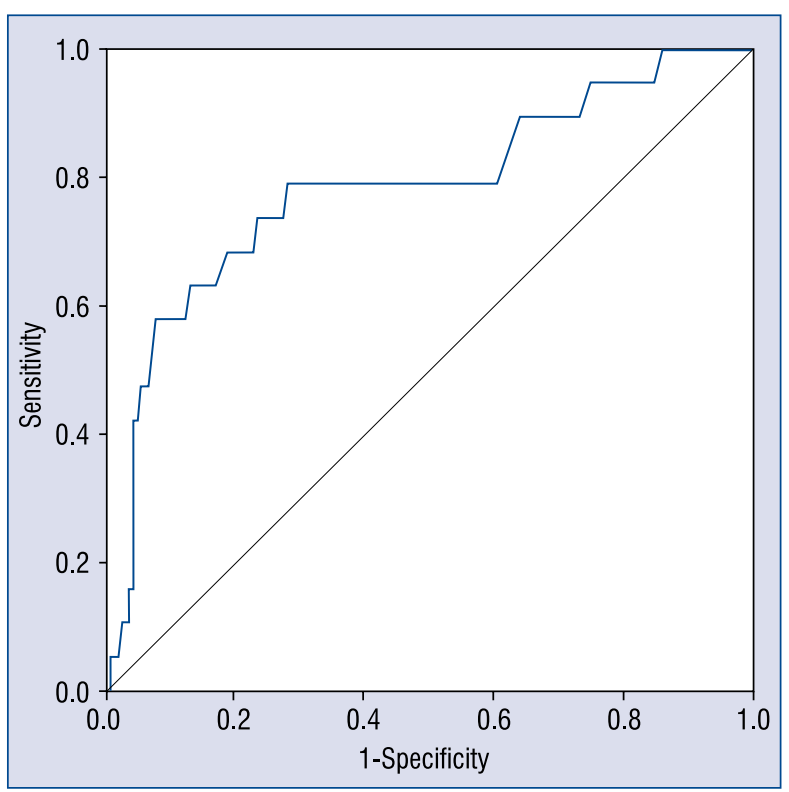

Figure 2. Receiver operating characteristic curve of blood urea nitrogen for predicting in-hospital mortality in patients with acute aortic dissection.

Urea is synthesized in liver via protein catabolism and blood urea is freely filtered at the glomerulus and undergoes tubular reabsorption. In the absence of enhanced protein catabolism, such as severe infection and burning, trauma, glucocorticoids therapy or high protein diet, urea levels in blood is determined by the GFR and tubular reabsorption. In the setting of $\mathrm{AAD}$, hemodynamic disturbances lead to activation of the neurohormonal axis, resulting in increased sympathetic nervous system [10] and renin-angiotensin-aldosterone system activity [11]. Increased angiotensin and adrenergic stimulation decrease renal urea excretion via effects on vascular, glomerular, and tubular effects on the kidney. These neurohormonal responses cause renal vasoconstriction, decreases in glomerular ultrafiltration, and increases in proximal tubular sodium and water reabsoption. Consequently, the fluid delivery in the collecting conduct will decrease and urine flow will slow. Because urea reabsorption in the distal tubule is urine flow dependent, the decreased slowing of tubular flow will enhance urea reabsorption [12]. Besides, AAD can cause low cardiac output by forming pericardial effusion or myocardial ischemia secondary to coronary artery involvement. In order to preserve systemic perfusion, arginine vasopressin (AVP) is released as a compensatory mechanism. AVP can rapidly increase urea permeability in the collecting duct through phosphorylation and apical plasmamembrane accumulation of the urea transporter A1 (UT-A1) and subsequently lead to increased reabsorption of urea in the collecting duct [13]. Therefore, increased BUN level could be regarded as a surrogate marker for hemodynamic and neurohormonal alternations in AAD.

The prognostic implication of increased BUN has been evaluated in patients with acute decompensated heart failure (ADHF). Studies have demonstrated that in the setting of ADHF, patients with high admission BUN level had low cumulative survival rate [14, 15]. In addition, Miura et al. [16] have shown that in patients admitted for ADHF, a BUN increase during hospitalization also indicated worse long-term prognosis, independent of renal function. Moreover, increased BUN is also associated with worse prognosis in a wide spectrum of patients with acute coronary syndromes $[1,17]$. However, few data are available on the relationship between BUN and clinical outcomes in patients with AAD. The present findings indicate for the first time that in patients with AAD receiving surgical or endovascular repair, high admission BUN levels were associated with high in-hospital mortality. BUN may be a promising marker for risk stratification in patients with AAD.

\section{Limitations of the study}

This study has several limitations. First, because of its observational nature, some factors that may have influenced the outcome of $\mathrm{AD}$ were unavailable, such as D-dimer and C-reactive protein. Second, this study was carried out in patients receiving surgical or endovascular repair for $\mathrm{AAD}$, excluding those receiving conservative treatment, which could limit results to extrapolate to a wider group of patients. Furthermore, this study was carried out in a single institution and enrolled a relatively small number of patients, which precluded subgroup analysis. A future prospective study with a larger sample size is required to confirm these findings.

\section{Conclusions}

In summary, the present study found that elevated BUN levels were an independent predictor of in-hospital mortality in patients with AAD receiving surgical or endovascular repair. Serum BUN may serve as a simple marker to identify high risk patients. 
Funding: This study was supported by a grant from the Natural Science Foundation of Zhejiang Province (LQ17H020005).

\section{Conflict of interest: None declared}

\section{References}

1. Kirtane AJ, Leder DM, Waikar SS, et al. TIMI Study Group. Serum blood urea nitrogen as an independent marker of subsequent mortality among patients with acute coronary syndromes and normal to mildly reduced glomerular filtration rates. J Am Coll Cardiol. 2005; 45(11): 1781-1786, doi: 10.1016/j.jacc.2005.02.068, indexed in Pubmed: 15936606.

2. Brisco MA, Coca SG, Chen J, et al. Blood urea nitrogen/creatinine ratio identifies a high-risk but potentially reversible form of renal dysfunction in patients with decompensated heart failure. Circ Heart Fail. 2013; 6(2): 233-239, doi: 10.1161/CIRCHEARTFAILURE.112.968230, indexed in Pubmed: 23325460.

3. Smith GL, Shlipak MG, Havranek EP, et al. Serum urea nitrogen, creatinine, and estimators of renal function: mortality in older patients with cardiovascular disease. Arch Intern Med. 2006; 166(10): 1134-1142, doi: 10.1001/archinte.166.10.1134, indexed in Pubmed: 16717177.

4. Klein L, Massie BM, Leimberger JD, et al. OPTIME-CHF Investigators. Admission or changes in renal function during hospitalization for worsening heart failure predict postdischarge survival: results from the Outcomes of a Prospective Trial of Intravenous Milrinone for Exacerbations of Chronic Heart Failure (OPTIMECHF). Circ Heart Fail. 2008; 1(1): 25-33, doi: 10.1161/CIRCHEARTFAILURE.107.746933, indexed in Pubmed: 19808267.

5. Ugajin M, Yamaki K, Iwamura N, et al. Blood urea nitrogen to serum albumin ratio independently predicts mortality and severity of community-acquired pneumonia. Int J Gen Med. 2012; 5: 583-589, doi: 10.2147/JJGM.S33628, indexed in Pubmed: 22866010.

6. Koutroumpakis E, Wu BU, Bakker OJ, et al. Admission Hematocrit and Rise in Blood Urea Nitrogen at 24h Outperform other Laboratory Markers in Predicting Persistent Organ Failure and Pancreatic Necrosis in Acute Pancreatitis: A Post Hoc Analysis of Three Large Prospective Databases. Am J Gastroenterol. 2015; 110(12): 1707-1716, doi: 10.1038/ajg.2015.370, indexed in Pubmed: 26553208.

7. Rhoney DH, Parker D, Millis SR, et al. Kidney dysfunction at the time of intracerebral hemorrhage is associated with increased in-hospital mortality: a retrospective observational cohort study.
Neurol Res. 2012; 34(5): 518-521, doi: 10.1179/1743132812Y.00 00000041, indexed in Pubmed: 22664363.

8. Takahashi T, Hasegawa T, Hirata N, et al. Impact of acute kidney injury on in-hospital outcomes in patients with DeBakey type III acute aortic dissection. Am J Cardiol. 2014; 113(11): 1904-1910, doi: 10.1016/j.amjcard.2014.03.023, indexed in Pubmed: 24837272.

9. Ko T, Higashitani M, Sato A, et al. Impact of Acute Kidney Injury on Early to Long-Term Outcomes in Patients Who Underwent Surgery for Type A Acute Aortic Dissection. Am J Cardiol. 2015; 116(3): 463-468, doi: 10.1016/j.amjcard.2015.04.043, indexed in Pubmed: 26026862.

10. Zhipeng Hu, Zhiwei W, Lilei Yu, et al. Sympathetic hyperactivity and aortic sympathetic nerve sprouting in patients with thoracic aortic dissection. Ann Vasc Surg. 2014; 28(5): 1243-1248, doi: 10.1016/j.avsg.2013.11.016, indexed in Pubmed: 24440187.

11. Li Y, Hu J, Qian H, et al. Novel findings: Expression of angiotensin-converting enzyme and angiotensin-converting enzyme 2 in thoracic aortic dissection and aneurysm. J Renin Angiotensin Aldosterone Syst. 2015; 16(4): 1130-1134, doi: 10.1177/1470320314549219, indexed in Pubmed: 25237166.

12. Schrier RW. Blood urea nitrogen and serum creatinine: not married in heart failure. Circ Heart Fail. 2008; 1(1): 2-5, doi: 10.1161/CIRCHEARTFAILURE.108.770834, indexed in Pubmed: 19808263.

13. Sands JM, Blount MA, Klein JD. Regulation of Renal Urea Transport by Vasopressin. Trans Am Clin Climatol Assoc. 2011; 122(122): 82-92.

14. Aronson D, Mittleman MA, Burger AJ. Elevated blood urea nitrogen level as a predictor of mortality in patients admitted for decompensated heart failure. Am J Med. 2004; 116(7): 466-473, doi: 10.1016/j.amjmed.2003.11.014, indexed in Pubmed: 15047036.

15. Fonarow GC, Adams KF, Abraham WT, et al. ADHERE Scientific Advisory Committee, Study Group, and Investigators. Risk stratification for in-hospital mortality in acutely decompensated heart failure: classification and regression tree analysis. JAMA. 2005; 293(5): 572-580, doi: 10.1001/jama.293.5.572, indexed in Pubmed: 15687312.

16. Miura M, Sakata Y, Nochioka K, et al. Prognostic impact of blood urea nitrogen changes during hospitalization in patients with acute heart failure syndrome. Circ J. 2013; 77(5): 1221-1228, indexed in Pubmed: 23392088.

17. Aronson D, Hammerman H, Beyar R, et al. Serum blood urea nitrogen and long-term mortality in acute ST-elevation myocardial infarction. Int J Cardiol. 2008; 127(3): 380-385, doi: 10.1016/j. ijcard.2007.05.013, indexed in Pubmed: 17765341. 